\title{
DOR TORÁCICA NÃO-CARDIOGÊNICA
}

\author{
Gerson Ricardo de Souza DOMINGUES ${ }^{1}$ e Joaquim Prado P. MORAES-FILHO²
}

\begin{abstract}
RESUMO - Contexto - Dor torácica não-cardiogênica ou dor torácica funcional é síndrome clínica com elevada prevalência no mundo ocidental, podendo estar presente entre $15 \%$ a $30 \%$ dos pacientes com coronariografias normais. Tem importante impacto na qualidade de vida dos pacientes e associa-se com considerável aumento da utilização dos serviços de saúde. Fontes de informação - Para esta revisão, foram utilizadas as seguintes bases de dados: Medline, the Cochrane Library, LILACS e livros nacionais. Das publicações dos últimos 5 anos foram selecionadas fontes relevantes como artigos originais, artigos de revisão, consensos, diretrizes e revisões sistemáticas de literatura com meta-análise. Publicações relevantes anteriores ao período de tempo analisado, foram também incluídas. Resultados - Foram incluídas 44 publicações, sendo 28 artigos originais, 12 trabalhos de revisão, 2 diretrizes, 1 meta-análise e 1 consenso. Conclusões - A dor torácica não-cardiogênica abrange a investigação do trato digestório, do aparelho musculoesquelético, do aparelho respiratório e de distúrbios psicológicos. O objetivo do tratamento é o alívio ou eliminação do sintoma e deve estar voltado para o principal mecanismo gerador. A base do tratamento é medicamentosa, entretanto, pode ser necessária intervenção de natureza psicológica e, nos pacientes com acalásia a terapia endoscópica ou cirúrgica. Considerando-se que a maioria dos pacientes apresentarão causas relacionadas ao esôfago, sendo as principais, a doença do refluxo gastroesofágico e distúrbios motores, as principais medicações utilizadas no controle da dor torácica não-cardiogênica são os inibidores da bomba de prótons e os antidepressivos tricíclicos. Recentemente, novas modalidades diagnósticas e também formas de tratamento, tais como, a injeção por endoscopia de toxina botulínica no esôfago e a hipnose, estão em investigação e algumas poderão ocupar lugar no cenário do tratamento destes pacientes.
\end{abstract}

DESCRITORES - Dor no peito. Refluxo gastroesofágico. Antidepressivos. Inibidores da bomba de prótons.

\section{INTRODUÇÃO}

Dor torácica não-cardiogênica (DTNC), também denominada dor torácica não-cardíaca, é a dor retroesternal do tipo anginoso cuja origem não é cardiogênica. Apresenta elevada prevalência: nos Estados Unidos, por exemplo, até um quarto da população adulta refere a queixa. Por outro lado, dentre os pacientes que se submetem a coronariografia para investigação de dor torácica $10 \%$ a $30 \%$ apresentam laudos normais ${ }^{(2)}$. Destes, muitos continuam incomodados pelo sintoma apesar de estarem conscientes do seu caráter benigno e da inexistência de doença cardíaca associada. A DTNC tem importante impacto na qualidade de vida dos pacientes e está associada a considerável aumento de utilização dos serviços de saúde ${ }^{(2)}$.

O completo entendimento do mecanismo fisiopatológico está por ser determinado. As causas da DTNC nem sempre são claras e sua origem pode estar relacionada ao trato digestivo, com destaque para a hipersensibilidade visceral ${ }^{(13)}$, mas também, pode estar associada às entidades que comprometem o aparelho musculoesquelético, aparelho respiratório e distúrbios psicológicos ${ }^{(36)}$. O Consenso Roma III considerou a aplicação do termo "dor torácica funcional de presumível origem esofágica” para aqueles pacientes com episódios de dor torácica retroesternal de qualidade visceral sem explicação aparente, tendo sido previamente afastadas a origem cardíaca e as hipóteses diagnósticas de doença do refluxo gastroesofágico (DRGE) e distúrbios da motilidade esofágica ${ }^{(16)}$. Recentemente, novas modalidades diagnósticas e esquemas terapêuticos estão em investigação e algumas poderão desempenhar papel importante papel no contexto clínico destes pacientes.

Para fins da presente atualização, foram investigadas fontes selecionadas como artigos de revisão, consensos, diretrizes e revisões sistemáticas de literatura com metaanálise. Foram consideradas as publicações dos últimos 5 anos nas seguintes bases de dados: MEDLINE, the Cochrane Library e LILACS. Outras publicações relevantes, algumas anteriores ao período de tempo analisado, foram também incluídas.

\section{EPIDEMIOLOGIA}

A prevalência da DTNC não é conhecida na maioria dos países latino-americanos mas, pelo fato da DTNC estar frequentemente associada à DRGE, convêm analisar a prevalência desta última. De fato,

Departamento de Clínica Médica da Universidade Gama Filho, Rio de Janeiro, RJ; ${ }^{2}$ Departamento de Gastroenterologia da Faculdade de Medicina da Universidade de São Paulo, São Paulo, SP.

Correspondência: Dr. Gerson Ricardo de Souza Domingues - Av. Ayrton Senna, 1850 - sala 224 - Barra da Tijuca - 22775-003 - Rio de Janeiro, RJ

E-mail: gersondomingues@grdgastro.com.br 
a DRGE é afecção de grande importância pela sua elevada e crescente prevalência na população e por determinar sintomas de intensidade variável, que se manifestam por tempo prolongado, podendo prejudicar consideravelmente a qualidade de vida do paciente ${ }^{(10)}$. No Brasil, MORAESFILHO et al. ${ }^{(26)}$ em estudo populacional empreendido em 22 cidades, onde 13.959 indivíduos foram entrevistados, determinaram a prevalência de DRGE, a partir da queixa típica de pirose e regurgitação em cerca de $12 \%$. Pelo fato de o estudo em pauta ter considerado apenas a ocorrência de pirose e regurgitação, é lícito supor que o número que exprime a prevalência seja expressivamente maior, alcançando a faixa de $20 \%$, caso tivessem sido consideradas na entrevista as manifestações extraesofágicas e atípicas.

$\mathrm{Na}$ Argentina, dentre as manifestações atípicas da DRGE a DTNC é a mais comum. Estudo populacional naquele país encontrou prevalência anual de DTNC de 23,5\%, distribuída de forma equitativa em ambos os sexos ${ }^{(6)}$. Nos Estados Unidos, o distúrbio afeta igualmente ambos os sexos, embora mulheres apresentem tendência a procurar com mais frequência o atendimento médico do que homens ${ }^{(23)}$. No sexo feminino, a maior prevalência da DTNC é encontrada em idades inferiores a 25 anos e na faixa etária entre 45 e 55 anos.

Quando comparados com um grupo de pacientes com dor torácica por doença isquêmica do coração, foi observado que os pacientes com DTNC são em geral mais jovens, consomem mais bebidas alcoólicas e cigarros, sofrem de ansiedade e persistem à procura de tratamento, mesmo quando o diagnóstico para a DTNC foi estabelecido ${ }^{(38)}$. A maioria dos pacientes é diagnosticada e tratada por médicos clínicos, com apenas 17\% dos pacientes encaminhados para o gastroenterologista ${ }^{(44)}$ (Figura 1).

\begin{tabular}{l|c|c|}
\hline & DTNC & Dor anginosa cardíaca \\
\hline Idade & $<$ & $>$ \\
\hline Sintomas típicos & $<$ & $>$ \\
\hline Álcool/fumo & $>$ & $<$ \\
\hline Ansiedade & $<$ & $>$ \\
\hline Compreensão/controle & $\mathrm{N}$ & $\mathrm{A}$ \\
\hline ECG repouso & & \\
DTNC = dor torácica não-cardíaca \\
ECG = eletrocardiograma \\
A = normal \\
A = anormal
\end{tabular}

FISIOPATOLOGIA. ETIOPATOGENIA

A fisiopatologia da DTNC não está completamente elucidada. Os fatores etiopatogênicos propostos incluem a DRGE, distúrbios motores esofágicos, propriedades mecanofísicas do esôfago, hipersensibilidade visceral, processamento central do estímulo esofágico alterado, atividade autonômica alterada e comorbidades psicológicas ${ }^{(13,39)}$ (Figura 2).

\section{Doença do refluxo gastroesofágico}

DRGE é a causa mais comum de DTNC. LOCKE et al. ${ }^{(23)}$ encontraram que a DTNC é mais comumente relatada

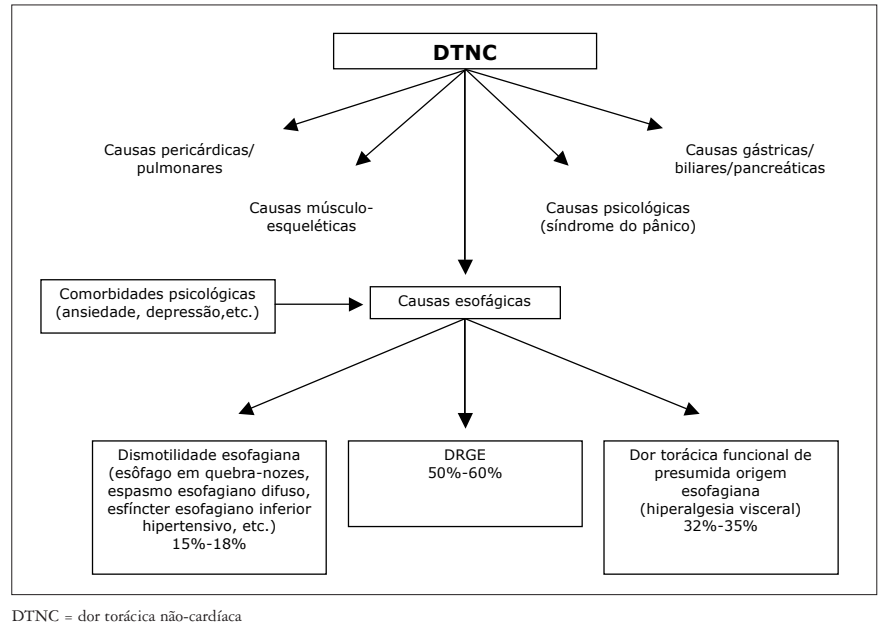

FIGURA 2. Etiopatogenia da DTNC ${ }^{(13,39)}$. Na prática clínica é muitas vezes difícil caracterizar o distúrbio predominante

por pacientes que apresentam pirose pelo menos uma vez por semana $(37 \%)$, quando comparados com aqueles que apresentam pirose infrequente $(30,7 \%)$ ou aqueles sem sintomas da DRGE (7,9\%). Os mecanismos determinantes para que um indivíduo com DRGE apresente pirose ou dor torácica estão, entretanto, por ser melhor estudados ${ }^{(39)}$.

Utilizando a pHmetria esofágica de 24 horas e a endoscopia digestiva alta, FASS et al. ${ }^{(1)}$ demonstraram que a DRGE pode ser caracterizada em até $60 \%$ dos pacientes com DTNC. Ainda que nesses pacientes a pHmetria esofágica possa apresentar resultados anormais em até cerca de $50 \%$ dos casos, somente $21 \%$ dos pacientes relataram episódio de dor torácica que coincidiu com o refluxo ácido, sugerindo que o índice de sintomas positivo é altamente indicativo de DTNC relacionada com o refluxo gastroesofágico ${ }^{(11)}$. Convém lembrar, entretanto, que este índice é influenciado pelo número de episódios de refluxo e pela frequência com que o paciente apresenta habitualmente a dor torácica, fatores que podem interferir na sensibilidade do mesmo. Por outro lado, a endoscopia digestiva isoladamente, revela a presença de esofagite erosiva em $10 \%$ a $25 \%$ dos casos de $\mathrm{DTNC}^{(39)}$. Até $80 \%$ desses pacientes com esofagite erosiva ou pHmetria anormal, responderão a vigoroso tratamento medicamentoso antirrefluxo, sugerindo que se um daqueles for positivo, a DRGE deve ser fortemente considerada como causa do sintoma ${ }^{(11)}$.

\section{Hipersensibilidade visceral}

Tem sido proposto que, a partir de estímulos gerados do tecido esofágico (como injúria tecidual, inflamação, espasmo muscular, estímulos mecânicos repetitivos) ou até mesmo estímulos fisiológicos, os nervos aferentes periféricos são sensibilizados, ativando mecanismos centrais e/ou periféricos, os quais, por sua vez, geram respostas exacerbadas a tais estímulos, caracterizando o fenômeno de hipersensibilidade visceral $^{(18)}$. Ao nível central, esta sensibilização ocorre no cérebro ou no corno dorsal da medula espinhal. A 
sensibilização periférica envolve redução do limiar de dor no esôfago e aumento do processo de transdução dos neurônios aferentes primários. Embora os eventos que promovem a duração prolongada da hipersensibilidade esofagiana ainda não estejam determinados, esta pode ser observada, mesmo após longos períodos decorridos do estímulo primário ou cicatrização da mucosa esofágica ${ }^{(13)}$.

Em pacientes com DTNC e em indivíduos controles saudáveis, SARKAR et al. ${ }^{(34)}$ realizaram a infusão de ácido clorídrico no esôfago distal durante 30 minutos. As respostas sensoriais foram monitorizadas no esôfago distal exposto ao ácido e no esôfago proximal não-exposto, ambos antes e após a infusão. No grupo controle, a infusão de ácido no esôfago distal reduziu o limiar de dor no esôfago proximal. Em contraste, os pacientes com DTNC já apresentavam a linha de base do limiar de dor mais baixa ab initio (quando comparados com o grupo controle). Após a infusão de ácido, o limiar de dor no esôfago proximal do grupo com DTNC reduziu-se ainda mais e por um período mais prolongado em relação ao grupo de indivíduos saudáveis. É interessante notar que a alodínia (sensação dolorosa causada por estimulação não-nociceptiva) secundária ${ }^{(39)}$ observada no esôfago proximal está, provavelmente, relacionada à sensibilização central. Pacientes com DTNC apresentam hipersensibilidade visceral e alodínia no esôfago.

Em estudo onde 14 pacientes com DTNC e 8 voluntários saudáveis, SARKAR et al ${ }^{(35)}$, empregando estimulação elétrica do esôfago proximal, demonstraram que o grupo de pacientes apresentava limiar mais baixo para percepção de dor torácica. Após 6 semanas de tratamento com inibidor da bomba protônica (IBP) (omeprazol $40 \mathrm{mg} / \mathrm{d}$ ), entretanto, foi documentado aumento do limiar de percepção da dor. Os resultados desse trabalho sugerem fortemente que a fisiopatologia da DTNC associada com a DRGE relacionase à hipersensibilidade visceral e, que esta, é responsiva a tratamento antissecretor.

Outros estudos utilizando diferentes metodologias, tais como distensão esofágica com balão ${ }^{(30)}$ e potencial evocado cerebral $^{(18)}$, têm confirmado o importante papel desempenhado pela hipersensibilidade visceral no desenvolvimento da DTNC, provavelmente, mais em função do resultado de amplificação pelo processamento cerebral do estímulo sensorial visceral do que resposta hiperálgica das vias viscerais aferentes ${ }^{(18)}$.

\section{Distúrbios motores esofágicos}

O papel dos distúrbios motores esofágicos como causa primária na geração da DTNC é controverso, já que apenas de $30 \%$ dos pacientes apresentam algum distúrbio motor. KATZ et al. ${ }^{(20)}$ e RENCORET et al. ${ }^{(32)}$ relataram que, dentre os pacientes com DTNC e com alterações motoras, o esôfago em quebra-nozes foi o distúrbio mais comum. LEMME et al. ${ }^{(21)}$ e HENRIQUEZ et al. ${ }^{(17)}$, entretanto, encontraram nos distúrbios motores inespecíficos e na hipotensão do esfíncter esofágico inferior, os distúrbios motores mais comumente identificados nestes pacientes.

RAO et al. ${ }^{(31)}$ estudaram distensões esofágicas progressivas com balão em um grupo de pacientes com DTNC e em um grupo controle de indivíduos saudáveis. Os pacientes que apresentaram dor torácica durante o teste foram reestudados após infusão venosa de atropina. Na maioria deles, as distensões do balão reproduziu a dor torácica em limiares mais baixos do que os do grupo controle, mesmo após o teste da atropina ter induzido o relaxamento da parede esofágica, sugerindo que, mais do que eventuais distúrbios motores, a causa da dor torácica funcional seria a hiperalgesia.

Em outro estudo foi observado que a maioria dos pacientes com DTNC e esôfago em quebra-nozes respondeu sintomaticamente ao tratamento antirrefluxo (com normalização da função motora esofágica ocorrendo numa minoria destes pacientes). Estes dados sugerem que a causa efetiva do sintoma estaria muito mais provavelmente relacionada com DRGE do que com as ondas peristálticas de amplitude elevada observadas no esôfago distal dos pacientes com esôfago em quebra-nozes ${ }^{(1)}$. Por outro lado, FORNARI et al. ${ }^{(14)}$ estudaram pacientes com esôfago em quebra-nozes controlados para a DRGE e com queixas de dor torácica e disfagia. A análise dos dados por regressão logística revelou associação significativa entre este distúrbio motor e os sintomas, sugerindo que o esôfago em quebra-nozes pode ser anormalidade motora importante na gênese da dor torácica e disfagia, e não apenas um mero achado manométrico.

A síndrome $X$, relativamente benigna, apresenta em seu quadro clínico, dor torácica típica com aspecto arteriográfico normal. Sua fisiopatologia envolve a redução do fluxo sanguíneo coronariano em resposta à perfusão ácida no esofago distal, o que sugere a presença de reflexo inibitório esofagocárdico(3). As contrações de longa duração da musculatura longitudinal, observadas pela ultrassonografia intraluminar de alta freqüuncia, parecem ser marcadores preditivos da dor torácica, mas é ainda pouco claro se essas contrações da musculatura fazem parte do mecanismo subjacente da dor torácica ou apenas representam um epifenômeno ${ }^{(23)}$.

\section{Comorbidades de natureza psicológica}

Em 17\% a 43\% dos pacientes com DTNC têm sido observados distúrbios de ordem psicológica, com elevada prevalência de síndrome do pânico (24\% a 70\%), ansiedade (33\% a 50\%) e depressão $(11 \% \text { a } 22 \%)^{(4)}$. Vale dizer que as comorbidades psicológicas e o estresse desempenham importante papel de aumentar a percepção ao estímulo intraesofágico.

\section{DTNC E QUALIDADE DE VIDA}

A DTNC pode afetar de forma significativa a qualidade de vida dos pacientes. É interessante observar que apesar de doença cardíaca já ter sido excluída por exames anteriores, ainda assim os pacientes frequentemente apresentam modificações e limitações de seu estilo de vida. Isto, muitas vezes agrava o quadro, pela persistência da crença de que têm doença cardíaca.

A DTNC implica em considerável custo devido ao absenteísmo do trabalho, uso crônico de drogas cardiovasculares, repetidas internações hospitalares para investigações e substancial ansiedade $^{(19)}$. 


\section{DIAGNÓSTICO E CONDUTA}

É importante destacar que a avaliação do esôfago de pacientes com DTNC está indicada somente após a investigação cardiológica ter efetivamente excluído a possibilidade de doença cardíaca como causa do sintoma ${ }^{(43)}$. Esta se impõe porque a análise isolada da apresentação clínica frequentemente não possibilita perfeita distinção entre dor torácica de origem esofágica e a de origem cardíaca ${ }^{(19)}$, e a morbidade e mortalidade das duas entidades são totalmente diferentes. A Figura 3 apresenta um algoritmo de manejo para pacientes com DTNC ${ }^{(13)}$

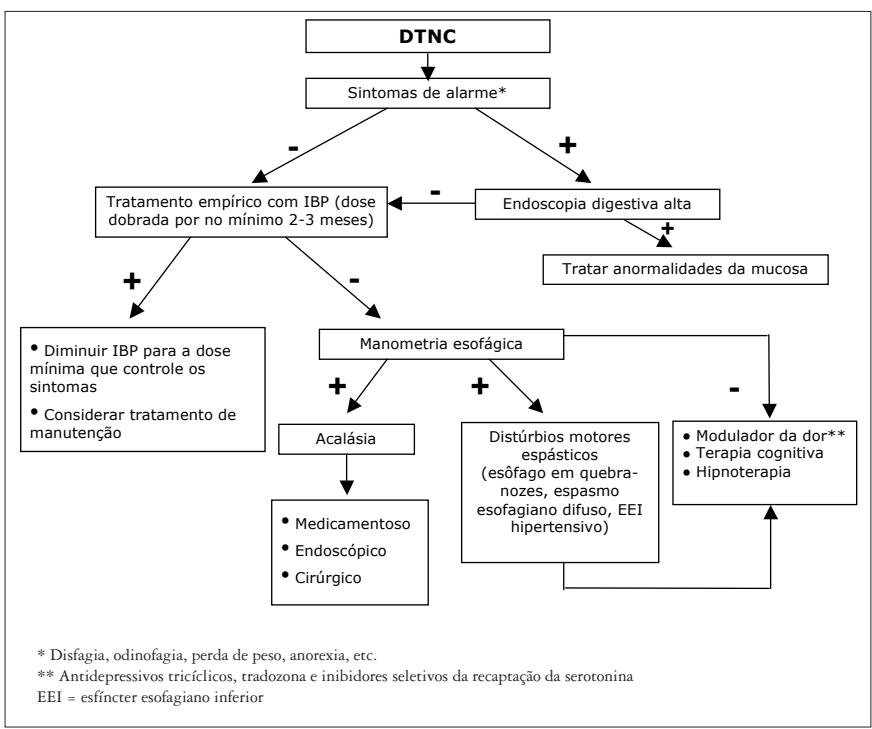

FIGURA 3. Algoritmo de manejo dos pacientes com $\mathrm{DTNC}^{(13,34)}$

\section{Teste terapêutico com inibidor da bomba protônica (IBP)}

O teste terapêutico com IBP é uma modalidade diagnóstica simples, de fácil acesso à população e que apresenta elevada sensibilidade. O teste consiste na aplicação de curso de IBP utilizando-se o dobro da dose padrão (Figura 4). O objetivo principal da prova é observar se ocorre melhora significativa do sintoma nos pacientes em um curto período de tempo. A sensibilidade do teste para o diagnóstico de DTNC relacionada com a DRGE varia entre $69 \%$ a $95 \%$ e sua especificidade, entre $67 \%$ e $86 \%{ }^{(42)}$. Pode ser realizado com qualquer um dos IBPs disponíveis no mercado e, em geral, com dose diária dobrada da medicação. Embora a duração do teste tenha variado entre 1 a 28 dias, o tempo médio mais frequentemente utilizado é de 7 dias $^{(43)}$. É considerado positivo quando a melhora da

\begin{tabular}{|l|c|}
\hline Omeprazol & 40 \\
\hline Lansoprazol & 30 \\
\hline Pantoprazol & 40 \\
\hline Rabeprazol & 20 \\
\hline Esomeprazol & 40 \\
\hline
\end{tabular}

FIGURA 4. Inibidores da bomba de prótons - dose padrão diária (mg) dor torácica for superior de $50 \%$ a $75 \%$ ou, eventualmente, valores superiores ${ }^{(43)}$.

Concluindo, em estudo de WANG et al. ${ }^{(41)}$ foi demonstrado que o uso do teste terapêutico com IBP para o diagnóstico da DRGE, em particular para os pacientes com DTNC, tem aceitável sensibilidade e especificidade e pode ser usado como abordagem inicial para a detecção da DTNC relacionada à DRGE.

\section{Manometria esofágica}

Pacientes que não responderam ao teste terapêutico com IBP e cuja pHmetria esofágica demonstrou efetivo controle do refluxo gastroesofágico ácido, ou seja, a DTNC não é dependente do refluxo ácido, têm indicação para a realização de manometria esofágica. Como já observado, este teste apresenta baixo rendimento diagnóstico, uma vez que a prevalência de distúrbios motores esofágicos como espasmo difuso esofágico e esôfago em quebra-nozes é baixa em pacientes com DTNC, sugerindo que a utilidade deste método diagnóstico é limitada ${ }^{(31)}$. É exceção, contudo, a possibilidade do diagnóstico de acalásia, que pode ter na dor torácica sua apresentação clínica atípica.

Resultados preliminares com um novo método para o diagnóstico dos distúrbios motores, a manometria esofágica de alta resolução, demonstraram que na investigação da DTNC, esta não oferece vantagens em relação à manometria convencional $^{(15)}$.

\section{3. pHmetria prolongada, pHmetria por telemetria e impedância esofágica}

Após a introdução do teste terapêutico com IBP, o papel da pHmetria esofágica de 24 horas tem se modificado nos últimos anos. Como visto acima, atualmente o teste tem sido empregado primariamente na investigação de pacientes com DTNC que não responderam ao teste terapêutico com $\mathrm{IBP}^{(42)}$. Nesses casos, a análise do índice de sintomas pode ser útil para aumentar a sensibilidade do teste, embora somente uma minoria de pacientes costumasse apresentar índice positivo, levando-se em conta que os relatos de dor torácica são relativamente incomuns durante a realização do teste.

Recentemente, PRAKASH e CLOUSE ${ }^{(28)}$, utilizando o sistema de pHmetria por telemetria (cápsula $\mathrm{Bravo}^{\circledR}$ ) com tempo estendido do estudo para 48 horas, demonstraram ganho diagnóstico, com aumento de $6,8 \%$ no número de pacientes que relataram sintomas durante o teste e de $100 \%$ no número de sintomas avaliados para a associação com um evento refluxo ácido. Em outro estudo ${ }^{(29)}$, os mesmos autores demonstraram que o sistema de pHmetria por telemetria foi capaz de aumentar a deteç̧ão de pacientes com DTNC e pHmetria anormal, com significativa probabilidade de associação entre este sintoma e o refluxo ácido.

O papel da impedância esofágica intraluminar em pacientes com DTNC permanece por ser elucidado ${ }^{(41)}$.

\section{Endoscopia digestiva alta}

O papel da endoscopia digestiva é limitado na investigação dos pacientes com DTNC devido à baixa frequência de 
achados na mucosa esofágica. É improvável que pacientes com DTNC não relacionada com a DRGE e que não apresentem sintomas de alarme, possam demonstrar achados endoscópicos significativos. Por outro lado, a avaliação endoscópica continua sendo importante para descartar a presença de esofagite péptica e/ou infecciosa (monilíase) e para descartar afecções de maior gravidade como esôfago de Barrett e câncer de esôfago ${ }^{(5,9)}$.

\section{Testes provocativos}

Os testes provocativos objetivam estimular a presença da queixa durante a realização dos mesmos. Infelizmente, contudo, testes como distensão por balão, edrofônio, perfusão ácida, ergonovina e betanecol, costumam apresentar baixa sensibilidade e, em certos casos, efeitos colaterais que impedem a sua realização de rotina como testes diagnósticos na prática clínica ${ }^{(13)}$.

\section{Avaliação psicológica}

Tendo em vista a elevada prevalência de alterações psicológicas que ocorrem neste grupo de pacientes, a decisão de submetêlos a avaliação e assistência psicológica especializadas deverá ser individualizada. Destaca-se, entretanto, que as principais indicações seriam os pacientes mais refratários às intervenções terapêuticas ou os que demonstram características, que sugerem a presença de distúrbio psicológico ${ }^{(42)}$. O primeiro passo nesse sentido deve ser a ênfase à natureza benigna da DTNC, já que esta atitude pode ser considerada como importante ação terapêutica preliminar. É certo, porém, que raramente os pacientes respondem somente a esta medida inicial, necessitando com frequência de auxílio de tratamento psicoterápico. A abordagem efetiva das comorbidades psicológicas deve, no entanto, ser reservada para os especialistas com experiência nesta área.

Nos pacientes com síndrome do pânico, o tratamento com alprazolam e clonazepam tem demonstrado redução da frequência dos ataques de pânico, episódios de dor torácica e escores de ansiedade ${ }^{(13)}$.

\section{TRATAMENTO}

O objetivo do tratamento é o alívio ou eliminação do sintoma e deve estar voltado para o principal mecanismo gerador. Assim, além das eventuais intervenções de natureza psicológica ou nos casos em que o diagnóstico é a acalásia, em que será aplicado o tratamento endoscópico e/ou cirúrgico, a natureza do tratamento é basicamente medicamentosa.

\section{Medicamentoso}

Os IBPs constituem a pedra angular para qualquer intervenção terapêutica na DTNC relacionada com a DRGE. Os pacientes devem receber inicialmente dose dobrada do IBP até a remissão do sintoma ou por, pelo menos, 2 meses $^{(33)}$, seguido de redução posológica até atingir a menor dose que os mantenha assintomáticos. Convém lembrar que como em outras manifestações atípicas da DRGE, pacientes com DTNC podem requerer mais de 2 meses de terapia para o adequado controle dos sintomas ${ }^{(33)}$. Em estudo duplo-cego, placebo-controlado, o omeprazol foi administrado na dose de $20 \mathrm{mg}$ em duas tomadas por dia, durante o período de 8 semanas, a pacientes com DTNC. Estes apresentaram significativa redução do número de dias com dor torácica e do escore de sua gravidade, quando comparados ao grupo placebo $^{(12)}$.

Os moduladores da dor ou analgésicos viscerais têm demonstrado que são capazes de melhorar de forma significativa a dor torácica de presumida origem esofágica não relacionada com a DRGE, como por exemplo, alterações espásticas ou por incoordenação da motilidade esofágica, com exceção da acalásia e hipersensibilidade visceral. Várias classes de fármacos têm sido avaliadas, incluindo os antidepressivos tricíclicos (ADT), trazodona, inibidores seletivos da recaptação da serotonina e teofilina ${ }^{(33)}$.

Os antidepressivos têm sido utilizados como moduladores da dor por quase 2 décadas no tratamento de pacientes com dor torácica de presumida origem esofágica, não relacionada com a DRGE. Os mecanismos pelos quais estes reduzem a dor visceral, entretanto, permanecem pouco claros. Alguns trabalhos sugerem sua ação por efeito central, enquanto outros, efeito periférico. Independentemente do sítio de ação, contudo, os ADT demonstram variada afinidade por receptores tais como acetilcolina, histamina 1 e $\alpha$-adrenégico ${ }^{(13)}$. Como os efeitos dos ADT variam segundo seus respectivos receptores, a falha de determinado ADT em melhorar os sintomas de determinado paciente não indica necessariamente falha futura com outro $\mathrm{ADT}^{(13)}$.

Os ADT incluem dentre outros, amitriptilina, imipramina e doxepin. Tem sido demonstrado que a imipramina aumenta o limiar de percepção esofágica para a dor em indivíduos normais, sem afetar o tônus esofágico, sugerindo efeito visceral analgésico. Efeito similar foi observado em pacientes com DTNC. Vale dizer que os ADT oferecem efeito relativamente duradouro para estes pacientes, embora seja necessária a interrupção da medicação em até $30 \%$ dos casos, decorrente do aparecimento de efeitos colaterais. O tratamento, portanto, deve ser iniciado com doses baixas ( $10 \mathrm{a} 25 \mathrm{mg}$ ), administradas ao deitar e, se necessário, com incrementos entre 10 a $25 \mathrm{mg}$ por semana, até atingir a dosagem de 50 a $75 \mathrm{mg} / \mathrm{dia}^{(8)}$.

A experiência com os inibidores seletivos da recaptação da serotonina é limitada, porém tem sido sugerido que esta classe de medicamentos, a exemplo dos ADT, exerça efeito neuromodulador mediando a percepção da dor visceral. VARIA et al. ${ }^{(40)} \mathrm{em}$ estudo duplo-cego, randomizado, placebocontrolado, em pacientes com DTNC, utilizaram sertralina em dose incial de $50 \mathrm{mg}$ por dia, ajustada posteriormente, conforme a resposta do paciente, até o máximo de $200 \mathrm{mg}$ por dia. Esses autores concluíram que os que receberam sertralina, apresentaram redução significativa do seu escore de dor torácica, quando comparados com aqueles que receberam placebo. Este estudo confirma o potencial papel dos inibidores seletivos da recaptação da serotonina no tratamento de pacientes com DTNC não relacionadas com a DRGE.

A trazodona, antidepressivo e ansiolítico, na dose de 100 a $150 \mathrm{mg}$, tem se mostrado eficaz no controle da 
DTNC não relacionada com a DRGE em pacientes com distúrbios motores espásticos sem, entretanto, afetar a amplitude das contrações esofágicas. CLOUSE et al.(7) em estudo duplo-cego, placebo-controlado, trataram um grupo de pacientes com DTNC e distúrbio motor esofágico com trazodona durante 6 semanas e compararam sua resposta com a de um grupo placebo. $\mathrm{O}$ grupo trazodona apresentou significativa melhora em relação ao grupo que utilizou placebo, sugerindo que aquele fármaco tem eficácia terapêutica nestes pacientes.

Alprazolam, na dose diária de $5 \mathrm{mg}$ e clonazepam, na dose diária de $1 \mathrm{mg}$ a $4 \mathrm{mg}$, se mostraram eficazes tanto na redução dos ataques de pânico como nos episódios de dor torácica de pacientes com DTNC e síndrome do pânico ${ }^{(13)}$.

O tratamento medicamentoso da DTNC com nitratos via sublingual ou via oral (longa duração), apresenta resultados conflitantes e, em sua maioria, está baseado em experiências limitadas e individuais. Por outro lado, o tratamento dos pacientes com DTNC e distúrbios motores com bloqueadores do canal de cálcio (diltiazem, nifedipina, verapamil, nimodipina e nisoldipina), que atuam relaxando a musculatura lisa, também parece ter valor limitado ${ }^{(16)}$. Além disto, seu uso pode ser complicado pelo desenvolvimento de efeitos colaterais como hipotensão, constipação e edema periférico.

Conquanto o uso da teofilina apresente contraindicação relativa em pacientes com DRGE, uma vez que tem efeito de relaxamento sobre o esfíncter esofágico inferior, esta pode ter papel no tratamento de pacientes com dor torácica funcional de presumida origem esofágica. Seu mecanismo envolve o bloqueio dos receptores de adenosina, porém estudos futuros são necessários para melhor definição do papel da teofilina no tratamento da $\mathrm{DTNC}^{(12)}$, particularmente em casos não relacionados com a DRGE.

Sildenafil é um potente inibidor do monofosfato de guanosina cíclica (GMP cíclico) específico da fosfodiesterase tipo V, que inativa o óxido nítrico estimulado pelo GMP. O acúmulo do óxido nítrico intracelular induz o relaxamento da musculatura lisa. A droga tem se mostrado benéfica em pacientes com esôfago em quebra-nozes e esfíncter inferior hipertenso por diminuir a amplitude das contrações no esôfago distal e prolongar a duração do relaxamento do esfíncter inferior. $\mathrm{O}$ uso de sildenafil tem utilização limitada na prática clínica em decorrência de seu custo elevado e efeitos colaterais. Não existem estudos com o sildenafil dirigidos especificamente a pacientes com $\mathrm{DTNC}^{(13)}$.

\section{Cirúrgico}

O sucesso da terapêutica cirúrgica antirrefluxo pela realização de fundoplicatura em pacientes com DTNC é incerto. Parece estar ligado à presença de um índice de sintomas positivo para a dor torácica relacionada ao refluxo ácido, ou seja, quando existe associação entre o episódio de dor torácica e o refluxo ácido na avaliação pré-operatória pela pHmetria esofágica. Nos casos em que a ocorrência do índice de sintomas é negativa, ou seja, existe fraca associação entre o refluxo ácido e o episódio de dor torácica, a melhora dos sintomas após tratamento cirúrgico decresce substancialmente ${ }^{(27)}$.
A realização da miotomia esofágica alongada com ou sem cirurgia antirrefluxo (fundoplicatura) nos pacientes com DTNC e distúrbios motores diferentes da acalásia permanece controverso e deve ser evitada ${ }^{(33)}$.

\section{Endoscópico}

A injeção de toxina botulínica no esfíncter esofágico inferior foi utilizada em alguns estudos não controlados, que incluíram pacientes com DTNC e distúrbios motores espásticos do esôfago. Em um deles houve redução de 50\% dos episódios de dor torácica em $72 \%$ dos indivíduos. No seguimento dos pacientes, a duração média de ausência da dor torácica foi de 7 meses. Infelizmente, $50 \%$ dos pacientes requereram segunda intervenção para a manutenção da remissão, como a repetição da aplicação de toxina botulínica $(43 \%)$, dilatação pneumática ou o uso de bugias ${ }^{(37)}$. Embora estes dados sugiram que a injeção de toxina botulínica em um percentual destes pacientes possa induzir melhora sintomática por curtos períodos de tempo, o valor desta modalidade terapêutica é limitado ${ }^{(22)}$. De forma semelhante, a dilatação pneumática utilizada no tratamento de pacientes com DTNC e distúrbios motores não relacionados à acalásia permanece controversa.

\section{Psicoterapia}

Em pacientes com ansiedade e síndrome do pânico pode estar indicado o tratamento psicoterápico e terapia comportamental cognitiva, realizados por profissional especializado. Estas opções terapêuticas induzem à melhora dos episódios de dor torácica e da qualidade de vida dos pacientes e, mesmo aqueles que não apresentam tais distúrbios, também podem se beneficiar desta modalidade terapêutica.

\section{Perspectivas futuras}

Futuros antagonistas da bomba ácida que exibam início rápido de ação, efeito dose-resposta previsível e profundo bloqueio à secreção ácida, poderão ter importante papel na DTNC relacionado com a DRGE como ferramenta diagnóstica ou como modalidade de tratamento a longo prazo.

A resposta ao tratamento com hipnose foi avaliada recentemente em estudo randomizado, placebo-controlado, em pequeno grupo de pacientes com DTNC com coronariografia e pHmetria esofágica normais. Cerca de $80 \%$ dos pacientes tratados com hipnoterapia, comparados a $23 \%$ do grupo controle, experimentaram melhora global significativa da dor torácica. Após 2 anos de seguimento do tratamento, os pacientes continuaram a melhorar dos sintomas com $93 \%$ destes apresentando também melhora no escore de qualidade de vida ${ }^{(25)}$. Este estudo preliminar sugere que a hipnose pode ser opção terapêutica não-medicamentosa no tratamento da DTNC, necessitando de estudos complementares para sua confirmação.

Em estudo inglês ${ }^{(24)}$, também de publicação recente, foi avaliado por meio de questionário, um grupo de pacientes com DTNC crônica, tendo a sua disposição após o tratamento clínico, a possibilidade de submeter-se a tratamento por acupuntura. Dentre aqueles que ainda apresentavam os sintomas, $42 \%$ 
demonstraram interesse nessa modalidade experimental de tratamento. Com base nestes dados, os autores propuseram que estudos devam ser realizados para avaliar a eficácia da acupuntura no tratamento destes pacientes.

Domingues GRS, Moraes-Filho JPP. Non-cardiac chest pain. Arq Gastroenterol. 2009;46(3):233-40.

ABSTRACT - Context - Non-cardiac chest pain or functional chest pain is a syndrome with high prevalence in ocidental world. Findings on $15 \%-30 \%$ of coronary angiograms performed in patients with chest pain are normal. Causes significant impact in quality of life of patients and is associated with increased use of the health care facilities. Data sources - To this review the following data base were accessed: Medline, the Cochrane Library, LILACS. The limit was the last 5 years publications and were selected relevant original articles, reviews, consensus, guidelines and meta-analysis. Results - Forty-four papers were selected, 28 original articles, 12 reviews, 2 guidelines, 1 consensus and 1 meta-analysis. Conclusions - Exclusion of cardiac disease is of crucial importance. On the other hand non-cardiac chest pain could be related to gastrointestinal, muscular and respiratory causes and/or psychological disturbances. Treatment aims to attack mechanism generator in order to relieve or to eliminate symptoms. Drugs are the cornerstone of treatment, exception to achalasia patients because those have better response to dilation of the esophagus or surgery, and to those who need intensive pyschological therapy. The most important drugs used are proton pump inhibitors and triciclic antidepressants, the latter, to modulate central signal process (visceral hypersensitivity) and autonomic response. Recently, new diagnostic facilities, and also therapeutic modalities, such as esophageal botulin toxin injection and hypnosis are under investigations. In the near future, maybe some of them would take a place in the therapeutic scenario of these patients.

HEADINGS - Chest pain. Gastroesophageal reflux. Antidepressive agents. Proton pump inhibitors.

\section{REFERÊNCIAS}

1. Achem SR, Kolts BE, Wears R, Burton L, Richter JE. Chest pain associated with nutcracker esophagus: a preliminary study of the role of gastroesophageal reflux. Am J Gastroenterol. 1993;88:187-92.

2. Chamber JB, Bass C. Chest pain with normal coronary anatomy: a review of natural history and possible etiologic factors. Prog Cardiovasc Dis. 1990;33:16184.

3. Chauhan A, Petch MC, Schofield PM. Cardiooesophageal reflex in humans as a mechanisms for "linked angina". Eur Heart J. 1996;17:407-13.

4. Cheng C, Wong WM, Lai CK, Wong BC, Hu WH, Hui WM, Lam SK. Psychological factors in patients with noncardiac chest pain. Psychosom Med. 2003;65:443-9.

5. Chinzon D, Hashimoto CL, Baba ER. Doença do refluxo gastroesofágico. In: Moraes-Filho JPP, editor. Tratado das enfermidades gastrintestinais e pancreáticas. São Paulo: Roca; 2008. p.499-515.

6. Chiocca JC, Olmos JA, Salis GB, Soifer LO, Higa R, Marcolongo M. Prevalence, clinical spectrum, and atypical symptoms of gastro-oesophageal reflux in Argentina: a nationwide population-based study. Aliment Pharmacol Ther, 2005;22:331-42.

7. Clouse RE, Lustman PJ, Eckert TC, Ferney DM, Griffith LS. Low-dose trazodone for symptomatic patients with esophageal contraction abnormalities. A doubleblind, placebo controlled trial. Gastroenterology. 1987;92:1027-36.

8. Clouse RE. Psychotropic medications for the treatment of functional gastrointestinal disorders. Clin Perspect Gastroenterol. 1999;2:348-56.

9. Cohen H, Moraes-Filho JPP, Cafferata ML, Tomasso G, Salis G, González O, Valenzuela J, Sharma P, Malfertheiner P, Armstrong D, Lundell L, Corti R, Sakai P, Ceconello I. An evidence-based, Latin-American consensus on gastrooesophageal reflux disease. Eur J Gastroenterol Hepatol. 2006;18:349-68.

10. Cury MS, Ferrari AP, Moraes-Filho JPP. Evaluation of the health-related quality of life in gastroesophageal reflux disease patients before and after treatment with pantoprazole. Dis Esophagus. 2006;19:289-93.

11. Fass R, Naliboff B, Higa L, Johnson C, Kodner A, Munakata J, Ngo J, Mayer EA. Differential effect of long term esophageal acid exposure on mechanosensitivity and chemosensitivity in humans. Gastroenterology. 1998;115:1363-73.

12. Fass R. Chest pain of esophageal origin. Curr Opin Gastroenterol. 2002;18:464-70.

13. Fass R, Navarro-Rodriguez T. Noncardiac chest pain. J Clin Gastroenterol. 2008;42:636-46.

14. Fornari F, Farré R, van Malenstein H, Blondeau K, Callegari-Jacques SM, Barros SG. Nutcracker oesophagus: association with chest pain and dysphagia controlling for gastro-oesophageal reflux. Dig Liv Dis. 2008;40:717-22.

15. Fox MR, Bredenoord AJ. Oesophageal high resolution manometry - moving from research into clinical practice. Gut. 2008;57:405-23.

16. Galmiche JP, Clouse RE, Bálint A, Cook IJ, Kahrilas PJ, Paterson WG, Smout AJ. Functional esophageal disorders. Gastroenterology. 2006;130:1459-65.

17. Henriques A, Csendes A, Rencoret G. Prevalencia de los diferentes trastornos motores primarios del esófago. Estudo prospectivo de 5440 casos. Rev Méd Chile. 2007; $135: 1270-5$
18. Hollerbach S, Bulat R, May A, Kamath MV, Upton AR, Fallen EL, Tougas G. Abnormal cerebral processing of oesophageal stimuli in patients with noncardiac chest pain (NCCP). Neurogastroenterol Motil. 2000;12:555-65.

19. Kachintorn U. How do we define non-cardiac chest pain? J Gastroenterol Hepatol. 2005;20:s2-s5.

20. Katz PO, Dalton CB, Richter JE, Wu WC, Castell DO. Esophageal testing of patients with noncardiac chest pain or dysphagia. Results of three years' experience with 1161 patients. Ann Intern Med. 1987;106:593-7.

21. Lemme EMO, Moraes-Filho JPP, Domingues G, Firman CG, Pantoja JA. Manometric findings of esophageal motor disorders in 240 Brazilian patients with non-cardiac chest pain. Dis Esophagus. 2000;13:117-21.

22. Lemme EMO. Distúrbios motores do esôfago. In: Moraes-Filho JPP, editor. Tratado das enfermidades gastrintestinais e pancreáticas. São Paulo: Roca; 2008. p.481-98.

23. Locke GR III, Talley NJ, Fett SL, Zinsmeister AR, Melton LJ 3rd. Prevalence and clinical spectrum of gastroesophageal reflux: a population-based study in Olmsted Country, Minnesota. Gastroenterology. 1997;112:1448-56.

24. MacPherson H, Dumville JC. Acupuncture as a potential treatment for noncardiac chest pain - a survey. Acupunct Med. 2007;25:18-21.

25. Miller V, Jones H, Whorwell PJ. Hypnotherapy for non-cardiac chest pain: longterm follow-up. Gut. 2007;56:1643.

26. Moraes-Filho JPP, Chinzon D, Eisig JN, Hashimoto CL, Zaterka S. Prevalence of heartburn and gastroesophageal reflux disease in the urban Brazilian population. Arq Gastroenterol. 2005;42:122-7.

27. Patti MG, Molena D, Fisichella PM, Perretta S, Way LW. Gastroesophageal reflux disease (GERD) and chest pain. Results of laparoscopic antireflux surgery. Surg Endosc. 2002;16:563-6.

28. Prakash C, Clouse RE. Value of extended recording time with wireless $p H$ monitoring in evaluating gastroesophageal reflux disease. Clin Gastroenterol Hepatol. 2005;3:329-34.

29. Prakash $\mathrm{C}$, Clouse RE. Wireless $\mathrm{pH}$ monitoring in patients with non-cardiac chest pain. Am J Gastroenterol. 2006;101:446-52.

30. Rao SS, Gregersen H, Hayek B, Summers RW, Christensen J. Unexplained chest pain: the hypersensitive, hyperreactive, and poorly compliant esophagus. Ann Intern Med. 1996;124:950-8.

31. Rao S, Hayek B, Summers RW. Functional chest pain of esophageal origin: hyperalgesia or motor dysfunction. Am J Gastroenterol. 2001;96:2584-9.

32. Rencoret G, Csendes A. Estudio manométrico del esófago en pacientes con dolor torácico no cardíaco. Rev Méd Chile. 2006;134:291-8.

33. Rhee PL. Treatment of noncaardiac chest pain. J Gastroenterol Hepatol. 2005;20:s18-s19.

34. Sarkar S, Aziz Q, Woolf CJ, Hobson AR, Thompson DG. Contribution of central sensitisation to the development of non-cardiac chest pain. Lancet. 2000;356:1154-9.

35. Sarkar S, Thompson DG, Woolf CJ, Hobson AR, Millane T, Aziz Q. Patients with chest pain and occult gastroesophageal reflux demonstrate visceral pain hypersensitivity which may be partially responsive to acid suppression. Am J 
Gastroenterol. 2004;99:1998-2006.

36. Spalding L, Reay E, Kelly C. Cause and outcome of atypical chest pain in patients admitted to hospital. J R Soc Med. 2003;96:122-5

37. Sujata V, Pullela SV, Parkman HP. Treatment of chest pain in patients with nonachalasia spastic esophageal motor disorders using botulinum toxin injection [abstract]. Am J Gastroenterol. 2001;96:s23

38. Tew R, Guthrie EA, Creed FH, Cotter L, Kisely S, Tomenson B. A long term follow-up study of patients with ischemic heart disease versus patients with nonspecific chest pain. J Psychosom Res. 1995;39:977-85.

39. Van Handel D, Fass R. The pathophysiology of non-cardiac chest pain. J Gastroenterol Hepatol. 2005;20(Suppl):s6-s13.

40. Varia I, Logue E, O'Connor C, Newby K, Wagner HR, Davenport C, Rathey K, Krishnan KR. Randomized trial of sertralina in patients with unexplained chest pain of noncardiac origin. Am Heart J. 2000;140:367-72.
41. Wang WH, Huang JQ, Zheng GF, Wong WM, Lam SK, Karlberg J, Xia HH, Fass R, Wong BC. Is proton pump inhibitor testing an effective approach to diagnose gastroesophageal reflux disease in patients with noncardiac chest pain? A meta-analysis. Arch Intern Med. 2005;165:1222-8.

42. Wong WM, Fass R. Extraesophageal and atypical manifestations of GERD. J Gastroenterol Hepatol. 2004;19:s33-s43.

43. Wong WM. Use of proton pump inhibitor as a diagnostic test in NCCP. J Gastroenterol Hepatol. 2005;20:s14-s17.

44. Wong WM, Beeler J, Risner-Adler S, Habib S, Bautista J, Fass R. Attitudes and referral patterns of primary care physicians when evaluating subjects with noncardiac chest pain - a national survey. Dig Dis Sci. 2005;50:656-61.

Recebido em 22/1/2009. Aprovado em 15/4/2009. 